Research Article

\title{
The Use of Traditional Chinese Medicine in Relieving EGFR-TKI-Associated Diarrhea Based on Network Pharmacology and Data Mining
}

\author{
Shuaihang Hu $\mathbb{D}^{1},{ }^{1,2}$ Wenchao Dan $\mathbb{D}^{1,2}$ Jinlei Liu ${ }^{1}{ }^{1,2}$ Peng $\mathrm{Ha}^{1,}{ }^{3}$ Tong Zhou, \\ Xinyuan Guo $\mathbb{1}^{4},{ }^{4}$ and Wei Hou $\mathbb{( I D}^{1}$ \\ ${ }^{1}$ Guang'anmen Hospital, China Academy of Chinese Medical Sciences, Beijing 100053, China \\ ${ }^{2}$ Beijing University of Chinese Medicine, Beijing 100029, China \\ ${ }^{3}$ University of Leicester, University Road, Leicester LE1 7RH, UK \\ ${ }^{4}$ Cancer Hospital Chinese Academy of Medical Sciences, Beijing 100021, China
}

Correspondence should be addressed to Wei Hou; houwei1964@163.com

Received 14 February 2021; Accepted 23 March 2021; Published 1 April 2021

Academic Editor: Akhilesh K. Tamrakar

Copyright (C) 2021 Shuaihang Hu et al. This is an open access article distributed under the Creative Commons Attribution License, which permits unrestricted use, distribution, and reproduction in any medium, provided the original work is properly cited.

In this study, the role of traditional Chinese medicine (TCM) in relieving epidermal growth factor receptor-tyrosine kinase inhibitor- (EGFR-TKI-) associated diarrhea was discussed by network pharmacology and data mining. Prediction of drug targets by introducing the EGFR-TKI molecular structures into the SwissTargetPrediction platform and diarrhea-related targets in the DrugBank, GeneCards, DisGeNET, and OMIM databases were obtained. Compounds in the drug-disease target intersection were screened by absorption, distribution, metabolism, and excretion parameters and Lipinski's rule in Traditional Chinese Medicine Systems Pharmacology. TCM-containing compounds were selected, and information on the property, taste, and meridian tropism of these TCMs was summarized and analyzed. A target-compound-TCM network diagram was constructed, and core targets, compounds, and TCMs were selected. The core targets and components were docked by AutoDock Vina (Version 1.1.2) to explore the target combinations of related compounds and evaluate the docking activity of related targets and compounds. Twenty-three potential therapeutic TCM targets for the treatment of EGFR-TKI-related diarrhea were obtained. There were 339 compounds acting on potential therapeutic targets, involving a total of 402 TCMs. The results of molecular docking showed good binding between the core targets and compounds, and the binding between the core targets and compounds was similar to that of the core target and the recommended drug loperamide. TCMs have multitarget characteristics and are present in a variety of compounds used for relieving EGFR-TKI-associated diarrhea. Antitumor activity and the efficacy of alleviating diarrhea are the pharmacological basis of combining TCMs with EGFR-TKI in the treatment of non-small-cell lung cancer. The core targets, compounds, and TCMs can provide data to support experimental and clinical studies on the relief of EGFR-TKI-associated diarrhea in the future.

\section{Background}

Primary bronchial lung cancer is the most common malignant tumor in the world, of which approximately $80-85 \%$ are non-small-cell lung cancer (NSCLC), and nearly $70 \%$ of NSCLC patients are in the locally advanced stage or have metastatic lesions at the primary diagnosis [1]. Although chemotherapy was once considered the first-line treatment for NSCLC, advancements in targeted gene research have given rise to epidermal growth factor receptor-tyrosine kinase inhibitors (EGFR-TKIs), such as erlotinib and gefitinib, to replace chemotherapy as the first-line therapy for NSCLC patients who are positive for the relevant driver genes $[2,3]$. The adverse reactions to EGFR-TKI are different from those to chemotherapy, which include myelosuppression, nausea, and vomiting; the administration of EGFRTKI is prone to causing rashes, diarrhea, and other adverse reactions, which have a negative impact on the patients' 
quality of life and can even lead to drug discontinuation. Therefore, it is essential to prevent and control adverse reactions during targeted therapy.

In clinical practice, common EGFR-TKI drugs are divided into three generations: the first generation includes gefitinib, erlotinib, and icotinib; the second includes afatinib; the third includes osimertinib. All these drugs can cause varying degrees of adverse reactions, and these common adverse reactions to EGFR-TKI treatment limit the application of EGFR-TKI; the mechanism by which these adverse reactions occur is not clear, but studies have shown that it is related to the secretion of chloride [4]. According to a previously published EGFR-TKI phase III clinical study, the overall incidence of diarrhea is 9.5-95.2\%. EGFR-TKI treatment should be suspended if Grade 2 diarrhea lasts more than 48 hours, and for Grade 3 or higher diarrhea, EGFR-TKI treatment should be stopped until the grade of diarrhea is reduced to 1 or below. If symptoms are not relieved in 14 days, the administration of EGFR-TKI should be discontinued. Loperamide is often used in the clinical treatment of severe diarrhea, but there are few studies on the corresponding precautionary measures [5].

TCM has a long history of use for the treatment of diarrhea and has advantages in treating EGFR-TKI-related diarrhea, because besides relieving diarrhea, it can regulate the gastrointestinal function of patients, increase appetite, and prevent the reoccurrence of diarrhea [6]. TCM combined with targeted drugs can enhance efficacy, reduce toxicity, and reverse drug resistance, improving the quality of life for patients. The mechanism of action for TCM mainly involves the regulation of the immune function of lung cancer patients, thereby enhancing the migration of $\mathrm{T}$ cells. At the same time, it can inhibit the proliferation of tumor cells and induce tumor cell apoptosis $[7,8]$.

The synergistic effect of drugs on the human body can be explored through the analysis of network topology and nodes. Andrew Hopkins combined systems biology, pharmacology, information networks, and computer science to put forward network pharmacology, which demonstrates mutual complex disease-gene-target-drug relationships by applying a professional visual network analysis software in the form of network signals, allowing these relationships to be analyzed through the topology of the network and nodes [9]. Due to the pharmacodynamic material basis of TCM, the mechanisms and toxicological effects of compounds containing TCM are still not clear; as the quality of medicinal materials is difficult to control, it is different to study them on an integral level compared to a molecular level [10]. As TCM is characterized by "multiple components, multiple targets, low affinity, low selectivity", and its effective constituents can be applied to various targeted diseases, it has obvious advantages in the treatment of multiple factors of a polygenic disease. Therefore, using network pharmacology to study multiple ways of regulating signaling pathways and to explain and explore how to effectively improve the efficacy of drugs is more in line with the ideals of TCM compounds. In this study, by screening drug and disease targets and using a variety of databases to screen compounds containing TCM, a target-compound-TCM relationship network was constructed. This network was then used to explore potential therapeutic targets and related compounds and identify TCMs that can relieve EGFR-TKI-associated diarrhea and have certain antitumor effects. These results can provide a scientific molecular basis for the clinical application of TCM in combination with EGFR-TKI. The specific process of the study is shown in Figure 1.

\section{Materials and Methods}

2.1. Screening of Drugs and Targets of the Disease. Through a literature search, the molecular formulae of gefitinib, erlotinib, icotinib, afatinib, and osimertinib were obtained. Their molecular structures were downloaded separately in SDF format using the PubChem database (pubchem.ncbi.nlm.nih.gov) and uploaded onto the SwissTargetPrediction (http://www.swisstargetprediction. ch) platform $[11,12]$, with species set to "Homo sapiens", to predict the target of drug action. The results from the Swiss TargetPrediction platform were supplemented by consulting the literature.

To obtain the targets of diseases related to diarrhea, a search through the GeneCards (http://www.genecards.org) [13], OMIM (omim.org) [14], DisGeNET (http://www. disgenet.org) [15], and DrugBank (http://www.drugbank. ca) [16] databases was performed using "diarrhea", "diarrhoea", and "diarrheas" as keywords. Drug and disease targets were converted into standard gene names through the UniProt database (https://www.uniprot.org/) [17]. The targets from the intersection of drug prediction targets and disease targets were obtained.

2.2. Screening Target-Related Compounds and Potential Therapeutic Targets. A search was conducted for the compounds related to the intersection targets through the database of Traditional Chinese Medicine Systems Pharmacology (TCMSP; http://tcmspw.com/index.php) [18] and filtered by absorption, distribution, metabolism, and excretion (ADME) parameters and Lipinski's rule [19-22]: oral bioavailability: $\geq 30 \%$; drug-likeness: $\geq 0.18$; the number of bonds which allow free rotation around themselves $\leq 10$; topological polar surface area $\leq 60 \AA^{2}$; and molecular weight: 180-500 Da.

As some of the data provided by the TCMSP database may be inconsistent with actual applications, compounds that were filtered out were checked one by one to supplement the related compounds. The screened compounds were mapped to the intersection targets of diseases and drugs, with targets not containing active ingredients excluded and potential therapeutic targets screened out, and a potential therapeutic target-compound network diagram was drawn using Cytoscape 3.7.2 [23]. Core therapeutic targets were screened out after performing an analysis of the characteristics of the network using a network analyzer.

2.3. Screening and Analysis of TCMs. The TCMSP database provided a list of TCMs that corresponded to the compounds, and the corresponding TCMs were screened out through the relevant compounds. To make the research 

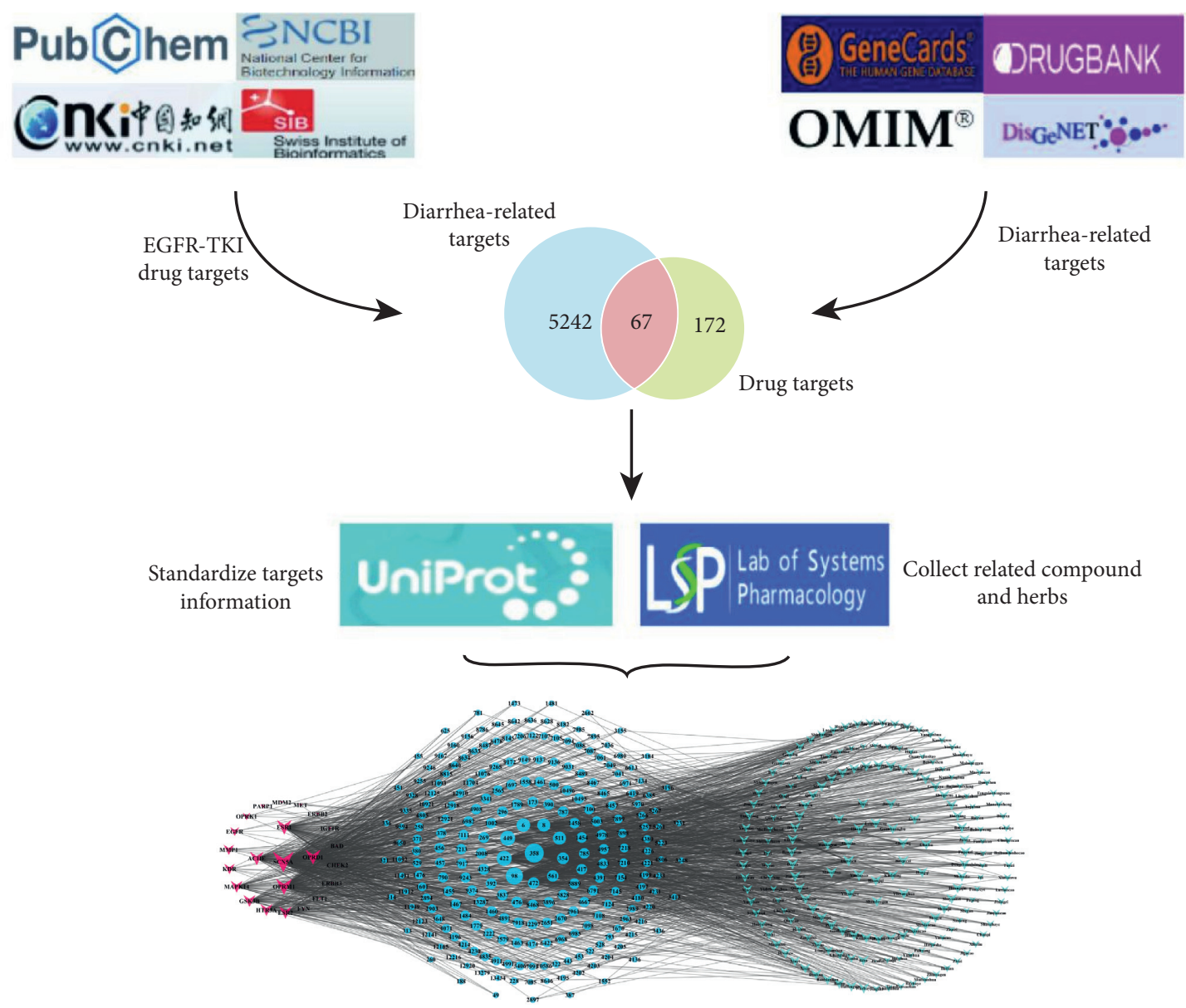

Targets-compounds-herbs network

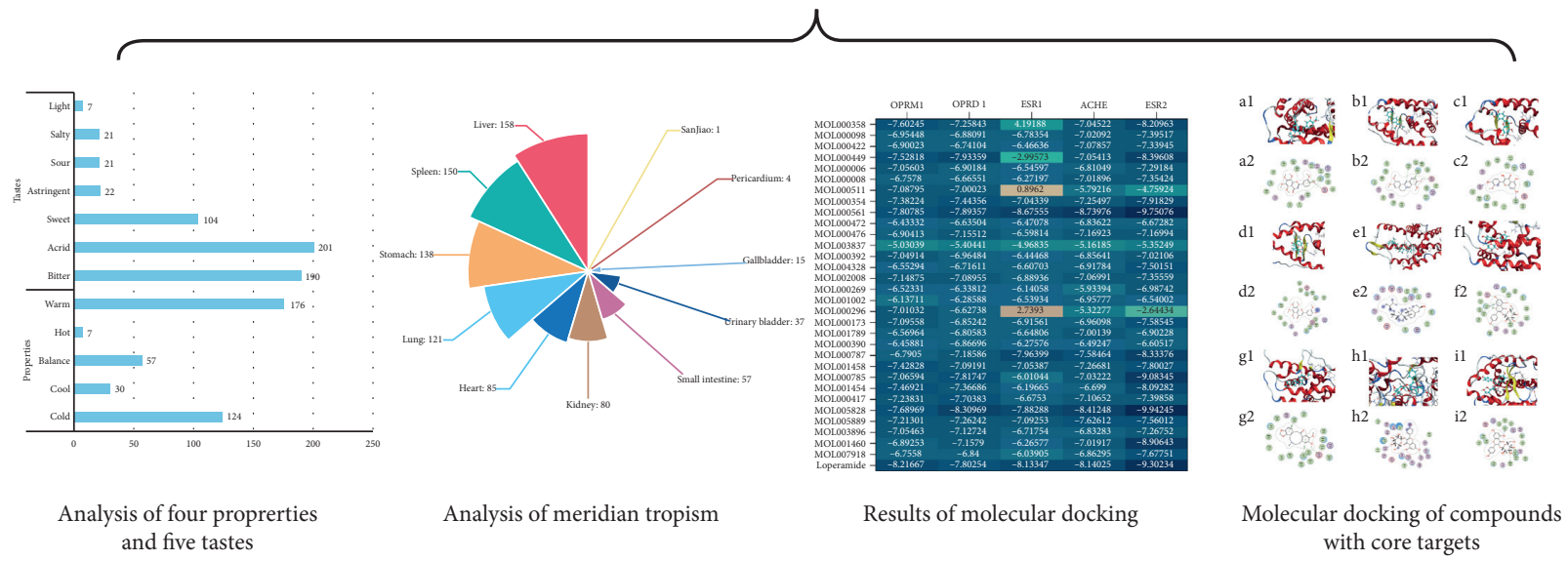

FIGURE 1: Framework of network pharmacology and data mining.

results standardized and practical, TCMs not listed in the "Pharmacopoeia of the People's Republic of China 2015 Edition" [24] were excluded. According to the "Pharmacopoeia of the People's Republic of China 2015 Edition" and the textbook "Chinese Pharmacy" in the "Thirteenth FiveYear Plan" [25], this study carried out frequency analysis on the components containing TCM.
2.4. Construction of Target-Compound-TCM Network. A target-compound-TCM network from the potential therapeutic targets and the corresponding compounds, as well as the TCMs contained in the candidate compound, was constructed using the software Cytoscape 3.7.2, and the network characteristics were analyzed by a network analyzer. The degree value indicates the number of connections a 
particular target has. The more related compounds there are in a single TCM and the more related targets the related compounds act on, the higher the degree value, based on the core compounds and TCMs selected [26].

\subsection{Molecular Docking between Target and Compound.} To confirm the credibility of the interaction between the core targets and the core components in the target-compoundTCM network and obtain a new drug-target combination, we selected five targets with a high median degree value in the target-compound-TCM network as receptors and used the recommended therapeutic drug, loperamide, as the ligand for molecular docking [6].

The crystal structures of the five selected targets were obtained from the Protein Data Bank (http://www.rcsb.org), a protein crystal database, and saved in PDB format. The 3D chemical structures of the candidate compounds were downloaded from the PubChem database and saved in SDF format; the output documents were converted to PDB format for subsequent molecular docking. The coordinate documents of the receptor and ligand were prepared using AutoDock Tools 1.5.6, with the water molecules deleted from the ligand, the ligand and receptor separated, nonpolar hydrogen added, and the Gasteiger charge calculated after it was saved in PDBQT format. The potential core ligand was subjected to the treatment of energy minimization, obtaining the ligand atom type after calculation, and finally saved in PDBQT format. The AutoDock Vina software was used for the calculation of docking of semisoft molecules. Compared with AutoDock4, AutoDock Vina 1.1.2 adopts a complex gradient algorithm and multithreading technology and performs molecular docking scoring to evaluate the matching degree and activity between the target and the ligand. A docking score of $<-4.25$ indicates binding activity between the ligand and the target, a score of $<-5.0$ indicates better binding activity, and a score of $<-7.0$ indicates strong docking activity [27].

\section{Results}

3.1. Drug-Disease Target Intersection. The probability value obtained from the SwissTargetPrediction platform to predict drug effect targets represents the probability that the query molecule targets the protein. From a total of 239 drug targets, the top 100, as ranked by probability, were selected after those with a probability of 0 were removed, and 5 drug targets were obtained after eliminating duplicates.

A total of 5245 diarrhea-related disease targets were obtained by searching the disease databases, merging the data, and removing duplicate values. A total of 67 targets were obtained from the intersection of drug and disease targets, as shown in Figure 2.

\subsection{Screening of Target-Related Compounds and Potential} Therapeutic Targets. A total of 67 targets were obtained from the intersection of EGFR-TKI and diarrhea and imported into the TCMSP database; 29 targets and 834 compounds of relevance were retrieved. After the selection of the ADME

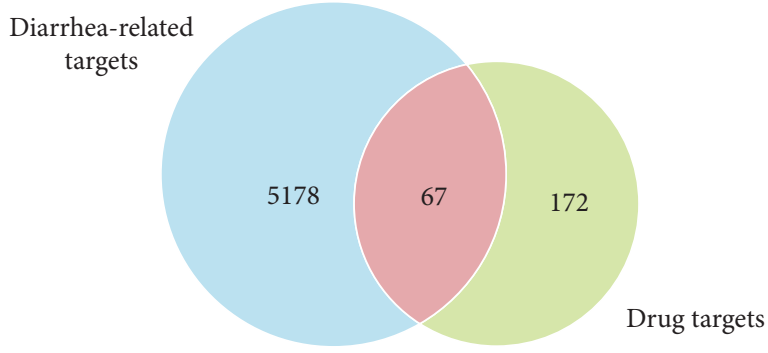

FIGURE 2: Intersection targets of EGFR-TKI and diarrhea.

parameters and Lipinski's rule, 293 compounds were obtained. The compounds excluded by the ADME parameters and Lipinski's rule were checked individually for the purpose of rigor, and 46 compounds associated with tumor treatment and adverse diarrhea effects were added, leading to a total of 339 compounds. There were 23 targets that met the 339 compounds at the intersection of EGFR-TKI and diarrhea; these were identified as the potential therapeutic targets for the treatment of adverse reactions of EGFR-TKI diarrhea with TCM (Table 1).

A network diagram of the final 339 compounds included with 23 potential therapeutic targets was constructed (Figure 3). To ensure a clear network diagram, the prefixes "MOL" and " 0 " of the compound name were deleted, leaving only the compound number. The network feature analysis was performed through a network analyzer plug-in, where the degree value determines the number of node connections and the role of the reaction nodes in the network is positively correlated with the target size in the diagram. Sodium channel protein type 5 subunit alpha (SCN5A), OPRM1, OPRD1, ESR1, ACHE, and ESR2 were core targets with large degree values of 280,158, 128, 121, 90, and 55, respectively. The compounds with large degree values all had multiple potential therapeutic targets, suggesting that the selection of the compounds corresponded to the ideals of TCM and had extensive therapeutic value.

3.3. Compounds Corresponding to TCM. The final 339 compounds included in the network diagram were imported into the TCMSP platform to obtain 432 kinds of TCMs contained in the compounds. Following the deletion of 30 kinds of TCMs that were not recorded in the "Pharmacopoeia of the People's Republic of China 2015 Edition", a total of 402 kinds of TCMs were incorporated.

The property, taste, and meridian tropism of the 402 TCMs were summarized and their frequency was analyzed. Acrid and bitter drugs had the highest frequencies, accounting for $35.51 \%$ and $33.56 \%$ of the selected TCMs, respectively. Drugs that were warm in property were the most frequently occurring, accounting for $44.67 \%$ of the selected TCMs, followed by those cold in property, which accounted for $31.47 \%$. The frequency analysis of meridian tropism showed that the frequency of liver, spleen, and stomach was the highest in the included TCMs, accounting for $52.71 \%$ of the total, while $14.3 \%$ were attributed to the lungs. It is evident that most of the TCMs included were 
TABLE 1: Potential therapeutic targets of TCM for the treatment of EGFR-TKI-related diarrhea.

\begin{tabular}{|c|c|c|}
\hline ID GeneSymbol & Uniprot ID & Protein name \\
\hline 1 SCN5A & Q14524 & Sodium channel protein type 5 subunit alpha \\
\hline 2 PARP1 & P09874 & Poly [ADP-ribose] polymerase 1 \\
\hline 3 OPRM1 & P35372 & Mu-type opioid receptor \\
\hline 4 OPRK1 & P41145 & Kappa-type opioid receptor \\
\hline 5 OPRD1 & P41143 & Delta-type opioid receptor \\
\hline 6 MMP1 & P03956 & Interstitial collagenase \\
\hline $7 \mathrm{MET}$ & P08581 & Hepatocyte growth factor receptor \\
\hline $8 \mathrm{MDM} 2$ & Q00987 & E3 ubiquitin-protein ligase $\mathrm{Mdm} 2$ \\
\hline 9 MAPK14 & Q16539 & Mitogen-activated protein kinase 14 \\
\hline $10 \mathrm{KDR}$ & P35968 & Vascular endothelial growth factor receptor 2 \\
\hline $11 \mathrm{IGF} 1 \mathrm{R}$ & P08069 & Insulin-like growth factor 1 receptor \\
\hline 12 HTR3A & P46098 & 5-Hydroxytryptamine receptor $3 \mathrm{~A}$ \\
\hline 13 GSK3B & P49841 & Glycogen synthase kinase- 3 beta \\
\hline 14 FYN & P06241 & Tyrosine-protein kinase fyn \\
\hline 15 FLT1 & P17948 & Vascular endothelial growth factor receptor 1 \\
\hline $16 \mathrm{ESR} 2$ & Q92731 & Estrogen receptor beta \\
\hline 17 ESR1 & $\mathrm{P} 03372$ & Estrogen receptor \\
\hline 18 ERBB3 & P21860 & Receptor tyrosine-protein kinase erbB-3 \\
\hline 19 ERBB2 & P04626 & Receptor tyrosine-protein kinase erbB-2 \\
\hline 20 EGFR & P00533 & Epidermal growth factor receptor \\
\hline $21 \mathrm{CHEK} 2$ & O96017 & Serine/threonine-protein kinase Chk2 \\
\hline $22 \mathrm{BAD}$ & Q92934 & Bcl2-associated agonist of cell death \\
\hline $23 \mathrm{ACHE}$ & P22303 & Acetylcholinesterase \\
\hline
\end{tabular}

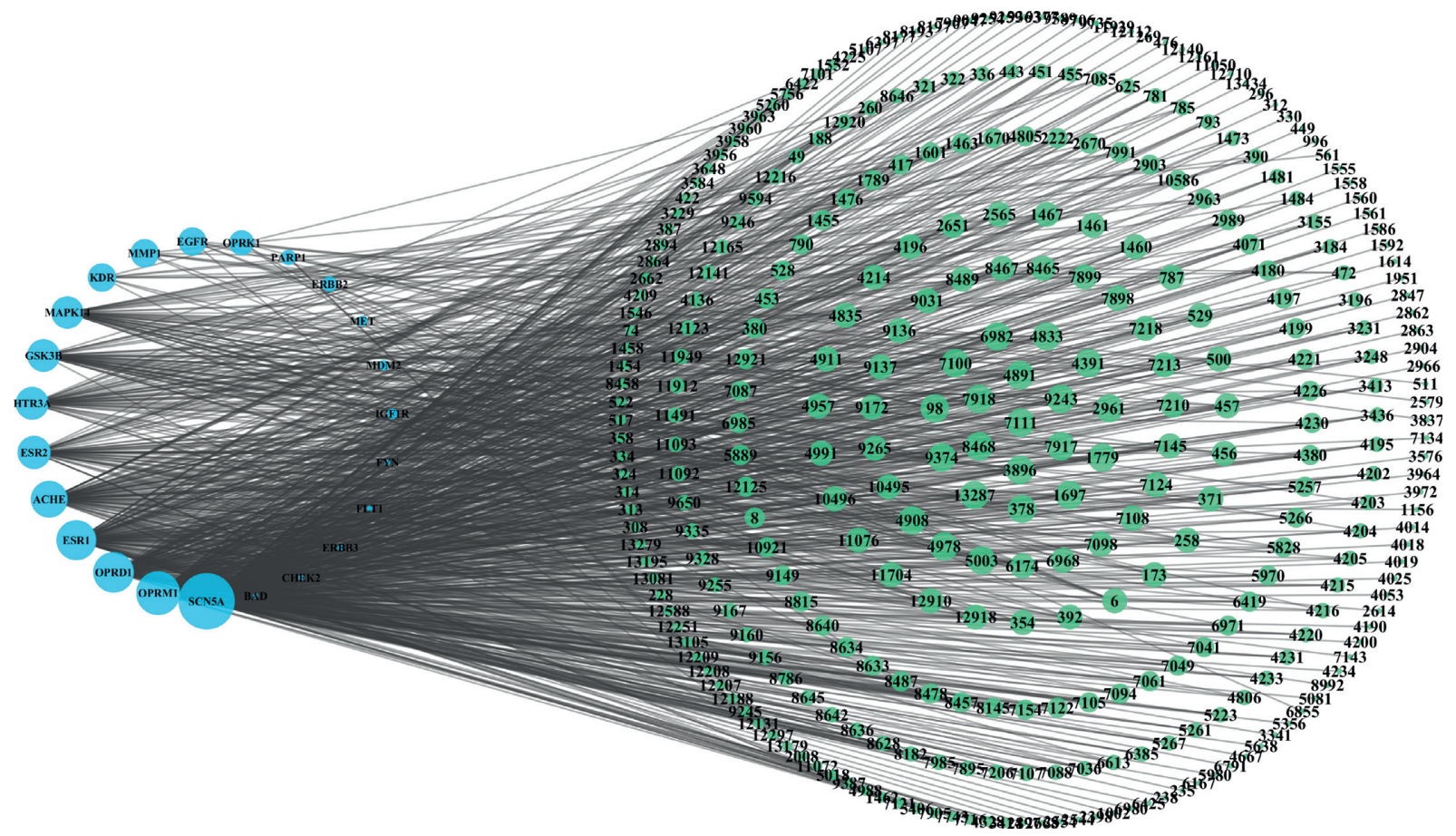

FIgURE 3: Target-compound network. (The network diagram consisted of 362 points and 1022 edges, with the potential therapeutic targets shown in blue and the compound targets shown in green.)

closely related to the liver, spleen, and stomach, followed by the lungs. These findings are consistent with the TCM theory of treating diarrhea and are related to the TCM theory of the digestive system (Figures 4 and 5).
3.4. Construction of the Target-Compound-TCM Network and Selection of Core Components. A network diagram of 23 potential therapeutic targets, 339 compounds, and 402 kinds of TCMs was constructed, with 764 nodes and 2707 edges. 


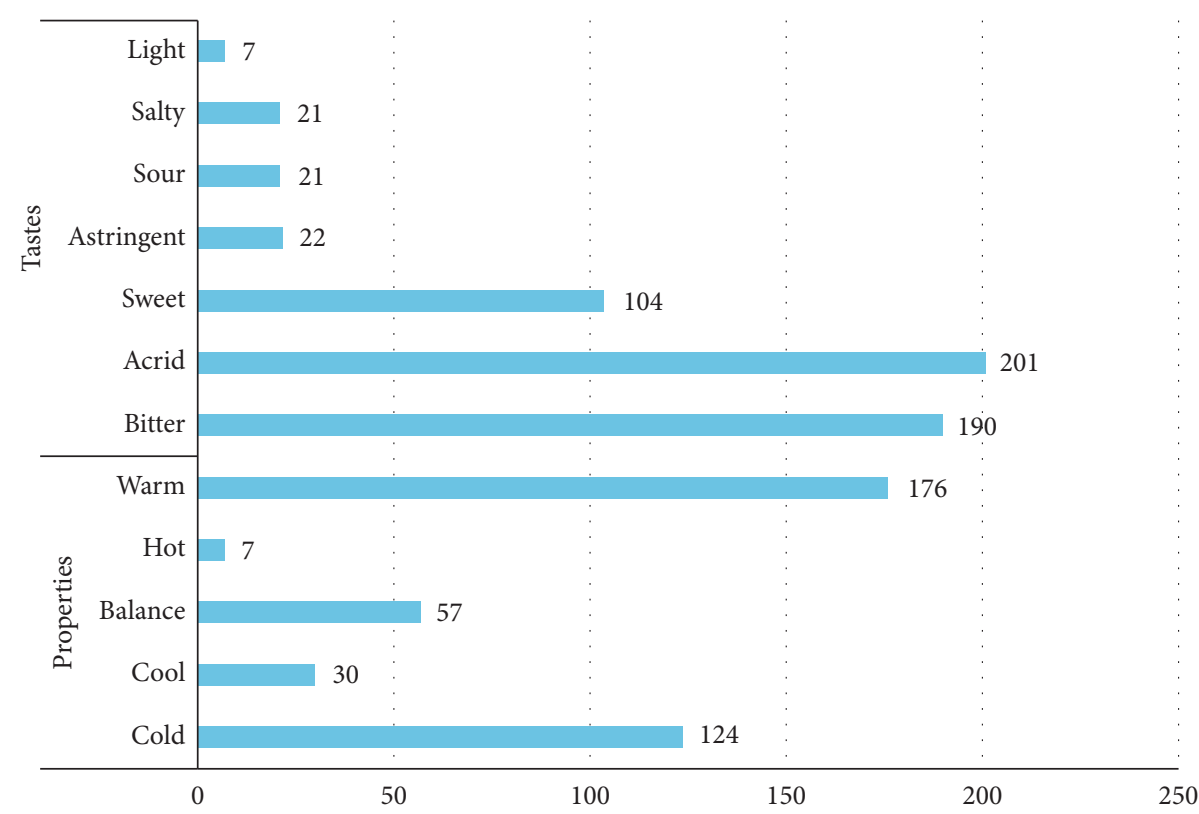

Figure 4: Analysis of four properties and five tastes.

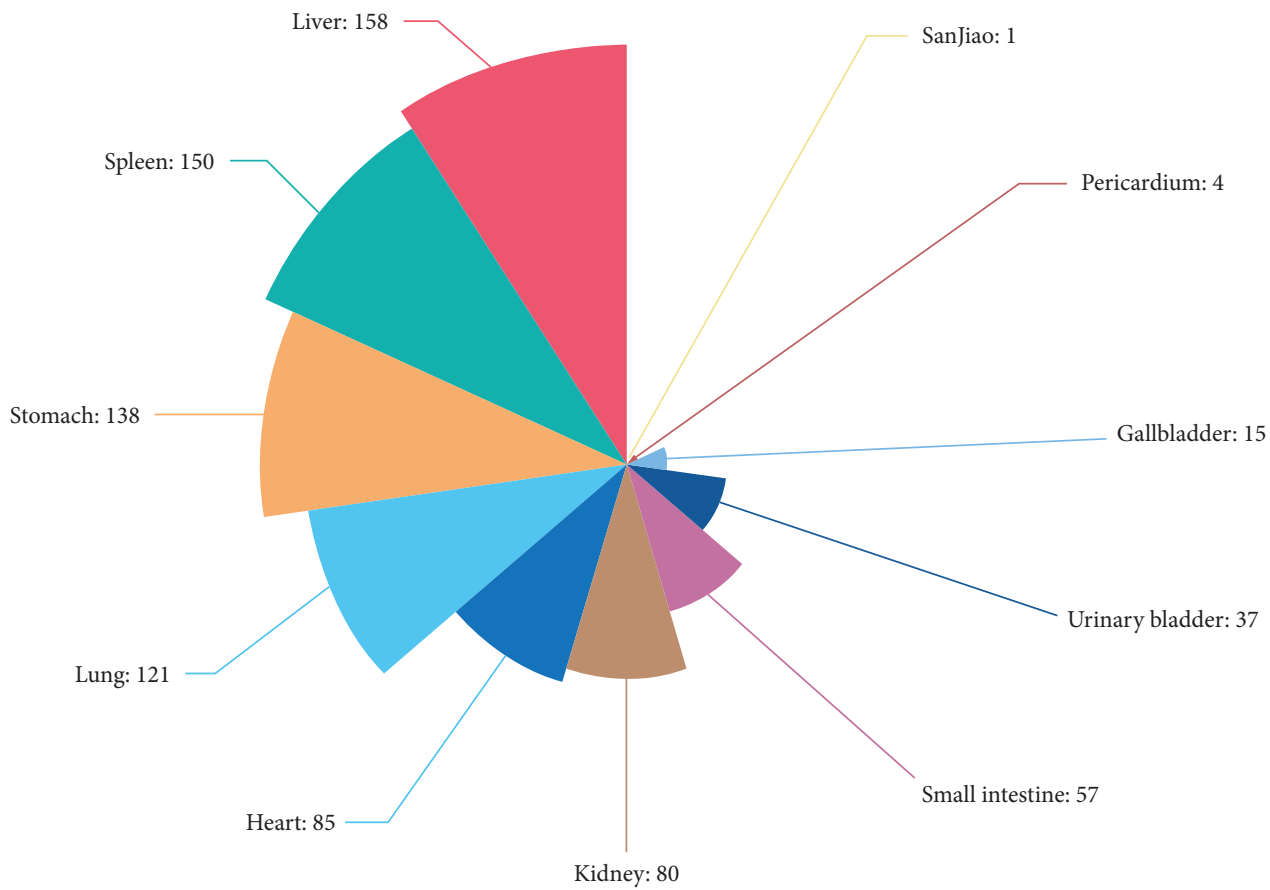

FIgURe 5: Analysis of meridian tropism.

For a clearer visual representation of the network relationships, only compounds and TCMs with degree values higher than the median (degree $\geq 4$ ) were kept in the network (Figure 6).

The top 10 TCMs based on degree value in the targetcompounds-TCM network diagram were Yanhusuo, Danshen, Gancao, Wuzhuyu, Huangbo, Guanhuangbo, Leigongteng, Baiqvcai, Gouteng, and Huangqi, as candidate compounds $38,28,26,16,16,13,13,12,12$, and 11 , respectively. Using the connection relationship between the related compounds and the targets in TCM, the potential targets of the TCMs for the treatment of EGFR-TKI-related diarrhea were collected. It was indicated that Yinyanghuo, Gaoliangjiang, Huangqi, Huzhang, Ziwan, Gancao, Jinqiaomai, Juhua, Yinxingye, and Heye were related to more targets, acting on $20,18,18,18,18,17,17,17,17$, and 16 targets, respectively, showing that these TCMs had a strong modulating function on diarrhea as an adverse effect of EGFR-TKI. The core compounds were selected with a median degree value of 4 and a standard of 8 , which was 


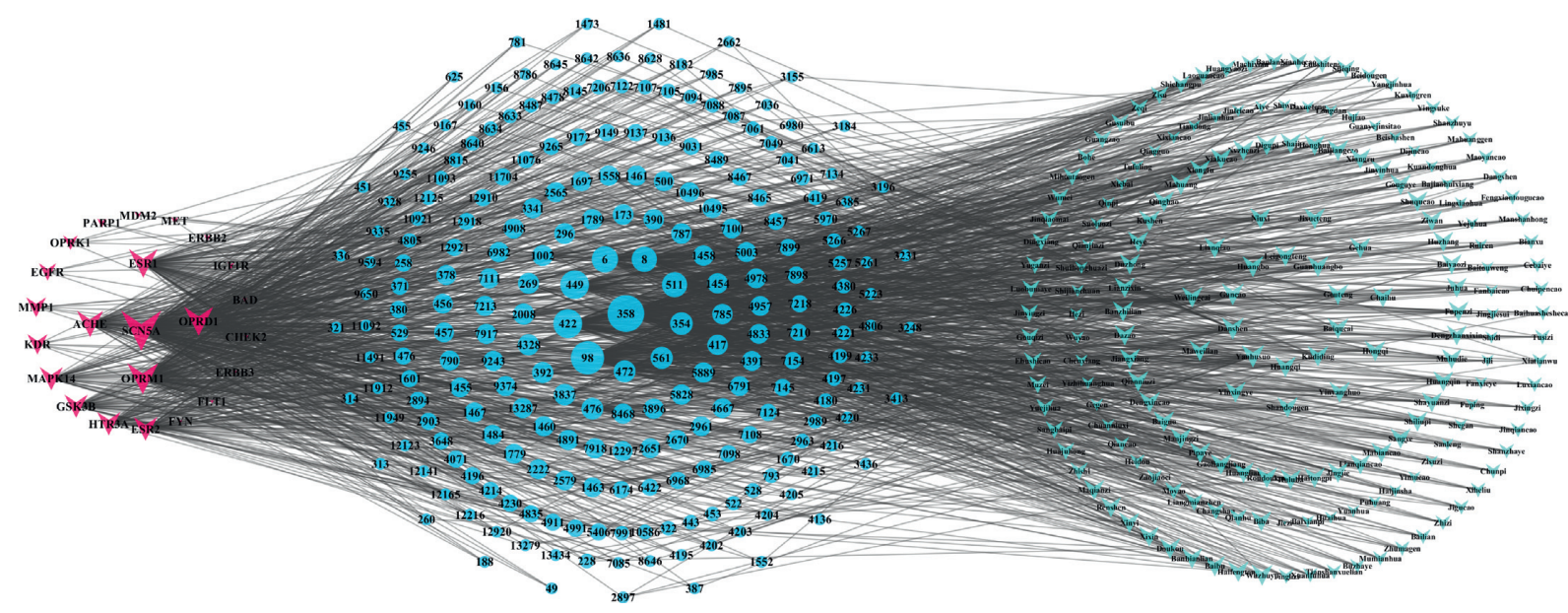

FIGURE 6: Target-compound-TCM network (compound degree $\geq 4$; TCM degree $\geq 4$; the network diagram consisted of 362 points and 1022 edges, with the potential therapeutic targets being shown in blue and the compound targets being shown in green).

greater than twice the median of the degree value. The top 10 compounds based on the degree value of the compounds were $\beta$-sitosterol, quercetin, kaempferol, stigmasterol, luteolin, apigenin, ursolic acid, isorhamnetin, astragalin, and emodin (Table 2).

3.5. Molecular Docking Results. The molecular docking of five core targets, OPRM1 (PDBID: 4DKL), OPRD1 (PDBID: 4N6H), ESR1 (PDBID: 1A52), ACHE (PDBID: 1ODC), and ESR2 (PDBID: 1L2J), with 31 core compounds and the commonly used therapeutic drug loperamide, resulted in a total of 186 receptor-ligand docking results (Figure 7). There were 38 combinations in the target-compound network with the highest docking score of $-9.9424 \mathrm{kcal} / \mathrm{mol}$ from MOL005828(nobiletin)-ESR2 and the lowest docking score of $-6.0104 \mathrm{kcal} / \mathrm{mol}$ from MOL000785(astragalin)-ESR1. The average score of $-7.0956 \mathrm{kcal} / \mathrm{mol}$ for the intranetwork combination implies good binding activity between the core targets and the core compounds, further proving the reliability of the therapeutic relationship between the compounds and the targets. Meanwhile, there were 117 new combinations outside the target-compound network. The top 3 combinations ranked according to affinity were MOL000561 (palmatine)-ESR2 with a score of $-9.7507 \mathrm{kcal} /$ mol, MOL001460(berberine)-ESR2 with a score of $-8.9064 \mathrm{kcal} / \mathrm{mol}$, and MOL000561(palmatine)-ACHE with a score of -8.7397 . The average score of the off-net combination was $-6.7672 \mathrm{kcal} / \mathrm{mol}$. The docking results showed that there were still drug-target combinations that were not included in the TCMSP, and the effects of the compounds in TCM on the adverse reactions to EGFR-TKI in the form of diarrhea need to be further studied. The core targets and docking of loperamide can be summarized as follows: $-8.2166 \mathrm{kcal} / \mathrm{mol}$ with OPRM1, $-7.8025 \mathrm{kcal} / \mathrm{mol}$ with OPRD1, $-9.3023 \mathrm{kcal} / \mathrm{mol}$ with ESR2, $-8.1334 \mathrm{kcal} / \mathrm{mol}$ with ESR1, and $-8.1402 \mathrm{kcal} / \mathrm{mol}$ with ACHE. This research has demonstrated that the potential therapeutic targets and drugs have good binding activity and have verified that the core targets selected in this study are of great significance for the treatment of diarrhea. The above-mentioned docking results offer reliable data support for further exploration of effective TCM ingredients and can serve as the theoretical reference for selecting effective targets and ingredients in future tests.

Taking into account the affinity values for molecular docking and the degree values of the target-compound-TCM network, the docking patterns with affinity $<-10$ in the target-compound-TCM network and the top 4 ranked by affinity values outside the network were presented as $2 \mathrm{D}$ and 3D molecular docking pattern plots. As shown in Figure 8, each ligand was inserted into the active pocket of the target and reacted with a number of residues of the target via hydrophobic interaction and hydrogen bond formation.

\section{Discussion}

At present, for NSCLC patients who are positive for EGFR gene mutations, using EGFR-TKI as the first-line treatment leads to better progression-free survival, especially in the Asia-Pacific region and Russia, where EGFR gene mutations are present in $49.3 \%$ of the total number of patients with NSCLC $[28,29]$. However, diarrhea is a common adverse event of EGFR-TKI drug use; if not taken correctly, it may lead to dehydration, forced reduction in drug dose, or even the interruption of treatment. The mechanism leading to such adverse reactions is not yet clear. It may involve imbalances in the ion transport system and abnormal secretion of chlorides, leading to secretory diarrhea [30], which lack effective preventive drugs. Although diarrhea is a common adverse reaction to all EGFR-TKIs, data show that secondgeneration inhibitors with a wider range of activities and targets cause a higher incidence of diarrhea [31]. Therefore, it is particularly important to explore the therapeutic targets related to EGFR-TKI and the drug-target relationship to investigate how EGFR-TKI may lead to adverse reactions such as diarrhea and to find effective therapeutic targets and drugs. Many studies have confirmed that TCM combined with EGFR-TKI treatment can improve the therapeutic effects and reduce toxicity by decreasing the incidence of 
TABLE 2: Core compounds (degree $\geq 8$ ).

\begin{tabular}{|c|c|c|c|}
\hline MolID & MolName & CAS & Degree \\
\hline MOL000358 & Beta-sitosterol & $83-46-5$ & 234 \\
\hline MOL000098 & Quercetin & $117-39-5$ & 185 \\
\hline MOL000422 & Kaempferol & $520-18-3$ & 128 \\
\hline MOL000449 & Stigmasterol & $83-48-7$ & 124 \\
\hline MOL000006 & Luteolin & $491-70-3$ & 91 \\
\hline MOL000008 & Apigenin & $520-36-5$ & 78 \\
\hline MOL000511 & Ursolic acid & $77-52-1$ & 77 \\
\hline MOL000354 & Isorhamnetin & $480-19-3$ & 43 \\
\hline MOL000561 & Astragalin & $480-10-4$ & 36 \\
\hline MOL000472 & Emodin & $518-82-1$ & 32 \\
\hline MOL000476 & Physcion & $521-61-9$ & 30 \\
\hline MOL003837 & Esculetin & $305-01-1$ & 26 \\
\hline MOL000392 & Formononetin & $485-72-3$ & 23 \\
\hline MOL004328 & Naringenin & $153-18-4$ & 23 \\
\hline MOL002008 & Myricetin & $529-44-2$ & 22 \\
\hline MOL000269 & Elemicin & $487-11-6$ & 20 \\
\hline MOL001002 & Ellagic acid & $476-66-4$ & 18 \\
\hline MOL000296 & Hederagenin & $465-99-6$ & 17 \\
\hline MOL000173 & Wogonin & $632-85-9$ & 15 \\
\hline MOL001789 & Isoliquiritigenin & $961-29-5$ & 15 \\
\hline MOL000787 & Fumarine & $130-86-9$ & 14 \\
\hline MOL000390 & Daidzein & $486-66-8$ & 14 \\
\hline MOL001458 & Coptisine & $3486-66-6$ & 13 \\
\hline MOL000785 & Palmatine & $3486-67-7$ & 12 \\
\hline MOL001454 & Berberine & $633-66-9$ & 12 \\
\hline MOL005828 & Nobiletin & $478-01-3$ & 11 \\
\hline MOL005889 & Rhamnetin & $90-19-7$ & 11 \\
\hline MOL000417 & Calycosin & $\begin{array}{l}20575-57- \\
\quad 9\end{array}$ & 11 \\
\hline MOL003896 & 7-Methoxy-2-methyl isoflavone & $\begin{array}{l}19725-44- \\
1\end{array}$ & 10 \\
\hline MOL001460 & Cryptopin & $482-74-6$ & 9 \\
\hline MOL004891 & Shinpterocarpin & $\begin{array}{l}157414-04- \\
5\end{array}$ & 9 \\
\hline MOL007918 & 2-Hydroxy-7-methoxy-1,8-dimethyl-5-ethenyl-9,10-dihydrophenanthrene & - & 9 \\
\hline MOL008468 & $\begin{array}{l}\text { Methyl (E)-2-[(2S, 3Z, 12bS)-3-ethylidene-2,4,6,7,12,12b-hexahydro-1h-indolo[3,2-h]quinolizin-2- } \\
\text { yl]-3-methoxyprop-2-enoate }\end{array}$ & - & 9 \\
\hline MOL000500 & Vestitol & $\begin{array}{l}20879-05- \\
\quad 4\end{array}$ & 8 \\
\hline MOL001461 & Dihydrochelerythrine & $6880-91-7$ & 8 \\
\hline
\end{tabular}

diarrhea as an adverse reaction [32-34], indicating that TCM has certain advantages in the treatment of diarrhea. It can also enhance the therapeutic effects, but the research on related drugs and molecular mechanisms is still insufficient and lacks specific TCM intervention in studies of EGFR-TKI use with diarrhea as an adverse reaction. Therefore, it is of great clinical significance to assess effective therapeutic ingredients and targets in TCM. This research used network pharmacology to explore the mechanisms behind multicomponent and multitarget TCMs, to identify effective therapeutic targets, related compounds, and related TCMs, and to provide data support for using TCM to further improve the therapeutic effect of EGFR-TKI and reduce the incidence of diarrhea.

4.1. Core Targets. After screening the potential therapeutic targets, those with a high degree 294 value were screened for certain antitumor and antidiarrhea effects, such as OPRM1 and OPRD1. Opioids that act through such targets can reduce gastrointestinal propulsive peristaltic contractions and increase circular muscle tension and intraluminal pressure [35]. Endogenous opioids maintain gastrointestinal homeostasis. The activation of intestinal opioid receptors such as $\mu$ receptors and $\delta$ receptors reduces epithelial secretion and increases water/electrolyte absorption, producing an antidiarrheal effect [36]. As a synthetic opioid receptor agonist, loperamide, a recommended drug for the treatment of diarrhea as an adverse reaction to EGFR-TKI, not only acts through $\mu$ receptors in the muscular plexus of the intestinal wall but also inhibits the release of acetylcholine, thus reducing intestinal peristaltic activity [37]. Antidiarrheal drugs based on such targets are under continuous development $[38,39]$. The neurotransmitter acetylcholine participates in various activities mainly through nicotinic and muscarinic receptors, of which $\mathrm{M} 3$ receptors are widely distributed in the gastrointestinal tract. It plays an 


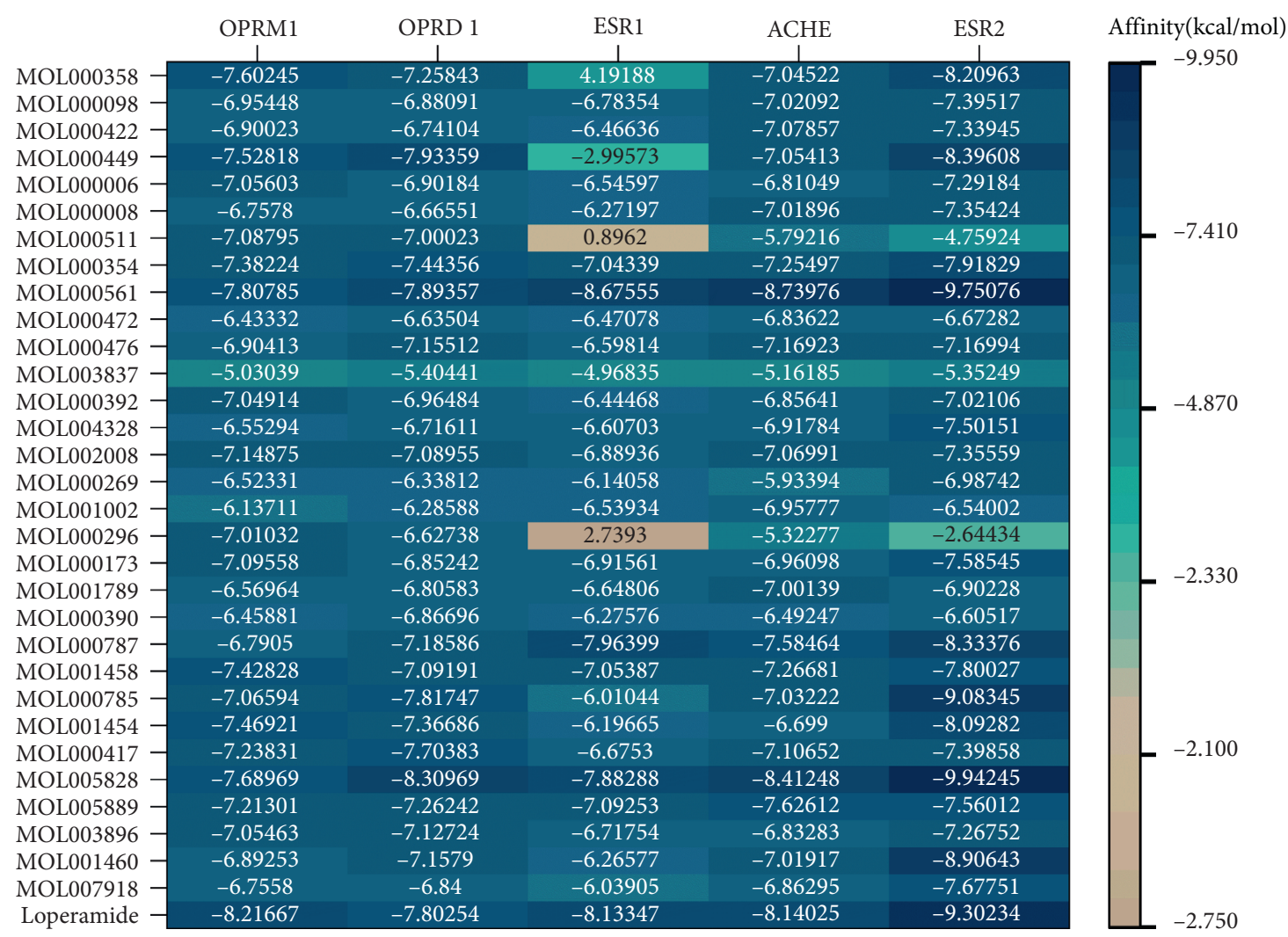

Figure 7: Results of molecular docking.

important role in maintaining the contraction of gastrointestinal smooth muscle [40]. ACHE, which is one of the core therapeutic targets identified in this study, can convert acetylcholine to choline and acetic acid, thereby preventing the contraction effect of acetylcholine on the smooth muscles of the gastrointestinal tract and producing a specific antidiarrheal effect. This feature has the same effect as loperamide in inhibiting the release of acetylcholine.

ESR1 and ESR2 are estrogen receptors, and studies have confirmed that there is a significant correlation between ESR1 expression and the overall survival of patients with NSCLC. Estrogen-induced growth of transplanted cells in vitro correlated closely with acute hormonal activation of mitogen-activated protein kinase signaling, and treatment with the antiestrogen drug Faslodex can reduce its growth. ESR1 expression is considered an independent prognostic factor for metastatic NSCLC [41, 42]. The single nucleotide polymorphisms of ESR2 are closely related to lung cancer in nonsmoking women [43], and studies have shown that nonsmoking women in Asia have a higher mutation rate of the EGFR gene [44]. We believe that ESR2 is correlated with EGFR's effect on metabolism during the treatment of NSCLC. However, at present, there are few studies on this kind of research, and its mechanism is not clear.

SCN5A is mainly responsible for the initiation and propagation of cardiac action potentials, thereby generating cardiac excitability and electrical stimulation to the cardiac muscles. Dysfunction of this target will cause excess sodium to enter the cardiomyocytes through abnormal channels, which leads to long QT syndrome [45]. Related studies have shown that QT interval prolongation is a relatively rare adverse event of several TKIs such as osimertinib and crizotinib, and it is believed that the heart should be monitored during the treatment of axitinib in patients with a previous family history of heart disease [46].

Mok et al. observed advanced NSCLC patients with the EGFR T790M mutation after an EGFR-TKI treatment of oral axitinib plus cisplatin or carboplatin. Among them, 4\% of patients taking osimertinib experienced QT interval prolongation, with $1 \%$ classified as having grade III adverse events [47]. At present, there are few studies on cardiotoxicity caused by TKIs. The SCN5A target identified in this study was predicted from the molecular structure of osimertinib on the SwissTargetPrediction platform. There were no such targets identified from other drug structures, so it is speculated that the adverse reaction of axitinib in prolonging QT interval may be related to this target, which provides new research ideas for the future exploration of TKI leading to adverse cardiac reactions.

4.2. Core Compounds. To better synergize with EGFR-TKI treatment, the selected compounds should not only alleviate the adverse reaction of diarrhea but also have an antitumor effect. Through topological analysis of the target-compoundTCM network, core compounds with higher degree values were identified and included $\beta$-sitosterol, quercetin, 

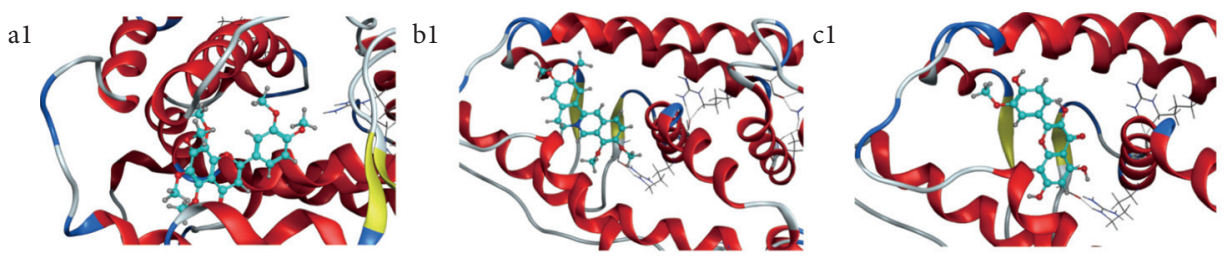

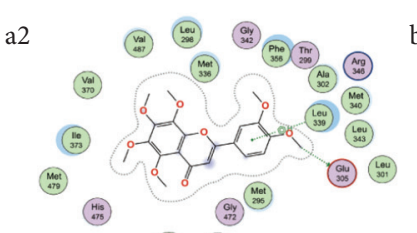

(iis) (iii)

d1

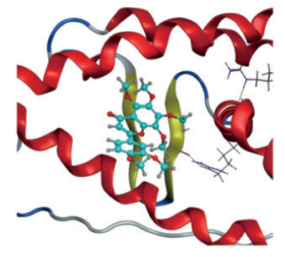

$\mathrm{d} 2$
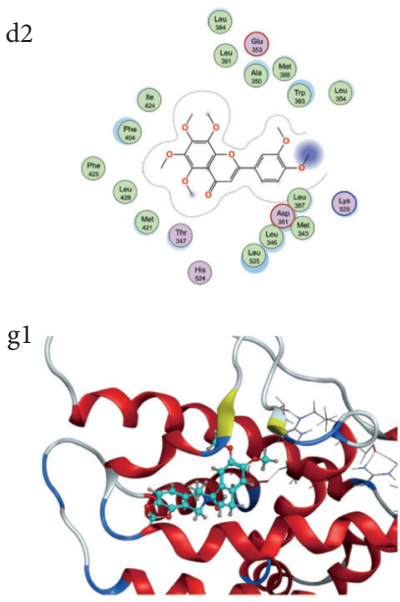

g2

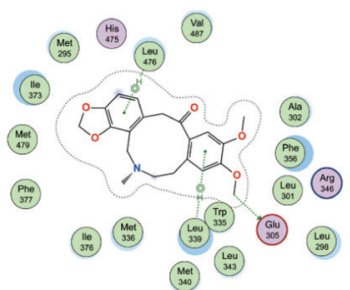

b2

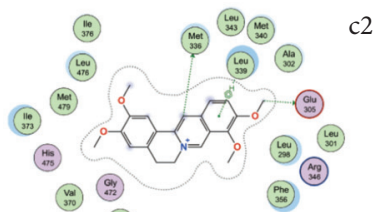

(iin)

e1

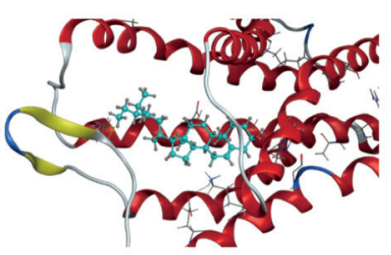

e2

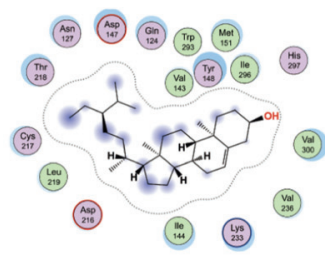

f2

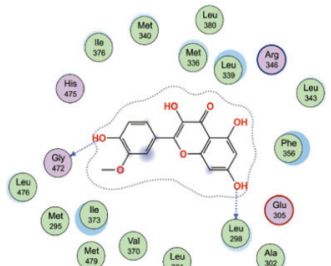

(iii: (iii) (iid)
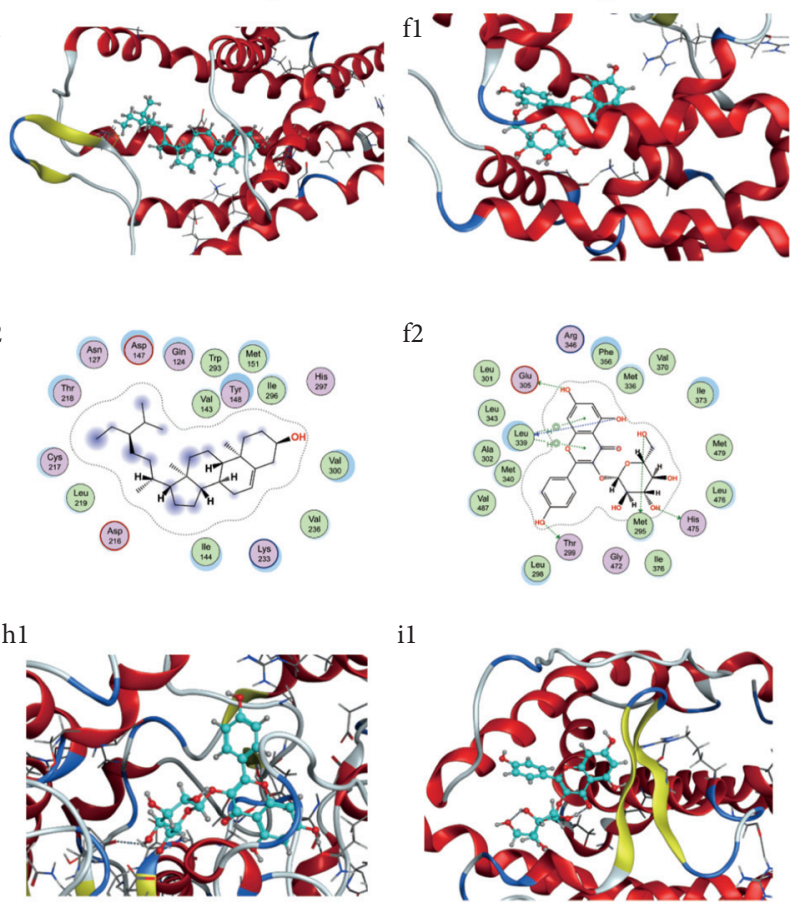

h2

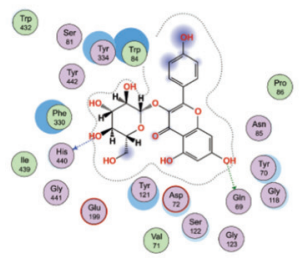

i2

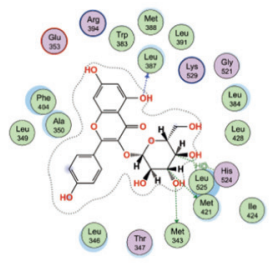

FIgURE 8: Molecular docking structure. (A) The overall 3D structure of the ligand-protein complex. The backbone of the protein was rendered as tubes and colored by chains. The ligands were rendered as sticks and colored in blue. (B) The 2D protein-ligand interaction diagrams of ligand-protein complexes. Protein residues were rendered as circles and colored based on their properties: green, hydrophobic residue; purple, polar residue (a: ESR2-nobiletin; b: ESR2-astragalin; c: ESR2-formononetin; d: ESR1-nobiletin; e: OPRM1-daidzein; f: ESR2-palmatine; g: ESR2-berberine; h: ACHE-palmatine; i: ESR1-palmatine).

kaempferol, stigmasterol, luteolin, and apigenin. All of them have antitumor effects and can relieve the adverse reaction of diarrhea to a certain extent.

$\beta$-Sitosterol, which is widely present in various plants [48], can interfere with various cell signaling pathways and affect the cell cycle, thereby inhibiting tumor cell proliferation. It has anticancer properties against breast, prostate, colon, lung, and gastric cancers [49]. $\beta$-Sitosterol can also inhibit the growth of A549 cells in a dose- and time-dependent manner, arresting the cell cycle of the tumor cells in the $\mathrm{G} 2 / \mathrm{M}$ phase. In vivo toxicity experiments show that $\beta$-sitosterol is nontoxic and reduces the absorption of cholesterol in the intestinal tract. It is a potential drug for the treatment of lung cancer [50]. 
Quercetin has various pharmacological properties such as having antitumor effects, conveying protection to the blood vessels, having anti-inflammatory and antioxidative properties, and being involved in immune regulation. Its main antitumor mechanism is related to the induction of apoptosis by inhibiting the cellular signal proteins regulated by antiapoptotic proteins, such as B-cell lymphoma-2 (Bcl2 ), and upregulating proapoptotic proteins. It also inhibits cell proliferation by blocking the progression of the cell cycle from G0/G1 to G2/M and inhibits angiogenesis and metastasis [51]. A study has shown that quercetin promotes the apoptosis of A549 cells by downregulating the expression of matrix metallopeptidase 9 (MMP-9) and transforming growth factor- $\beta$ (TGF- $\beta$ ) [52]. Li et al. [53] used various concentrations of quercetin and gemcitabine to treat lung cancer alone or in combination and found that quercetin, when combined with gemcitabine treatment, could act as a chemosensitizer by downregulating heat shock protein 70 (HSP70). However, its poor water solubility and low bioavailability limit further clinical use [54]. Phycophyllin also has good antitumor activity, and as the most active antidiarrheal ingredient in guava, it can inhibit the invasion of gastrointestinal diseases caused by Escherichia coli and Shigella flexneri in HEp-2 cells. Its anti-inflammatory and antioxidative effects can eliminate oxygen free radicals in the intestinal tract, reduce the loss of intestinal water and electrolytes, inhibit intestinal peristalsis caused by inflammation, and restore normal intestinal mechanical activity $[55,56]$.

Kaempferol can block the cell cycle through a variety of mechanisms, thus inhibiting the proliferation of cancer cells. It can inhibit the concentration of cyclin-dependent kinase (CDK), arresting cells in the G2/M phase [57]. The overexpression of the cell myelomatosis virus oncogene $(\mathrm{cMyc})$ in most cancers leads to excessive cell proliferation. Kaempferol can reduce the level of the cMyc mRNA and increase the level of the CDKN1A mRNA in cancer cells. The combination of cyclin-dependent kinase inhibitor 1A (CDKN1A) and the CDK complex inactivates CDK and blocks the cell cycle [58]. Kaempferol inhibits the secretion of vascular endothelial growth factor (VEGF) and inhibits angiogenesis [59]. Kaempferol and quercetin have synergistic antiproliferation effects on cancer cells through the decrease of the nuclear proliferating antigen Ki67 [60]. Inflammation is closely related to the occurrence and development of acute and chronic diarrhea. Kaempferol can regulate the activity of proinflammatory enzymes and inhibit transcription factors, adhesion molecules, and inhibit the expression of proliferation-related genes. At present, as the mechanisms behind diarrhea caused by EGFR-TKI are not clear, kaempferol, with the antitumor and anti-inflammatory properties, can provide a reference for the development of new drugs to alleviate the adverse reaction of diarrhea and strengthen EGFR-TKI therapy [61].

The other core components, such as stigmasterol, luteolin, apigenin, and ursolic acid, also have antitumor and anti-inflammatory effects. Stigmasterol inhibits cancer cell metastasis and arrests cell cycle in the G2/M phase [62]. It has also been shown to significantly decrease the expression of cyclooxygenase-2 (COX-2) and colony-stimulating factor- 1 , which could reduce the severity of colitis [63]. Luteolin can effectively inhibit the proliferation of NSCLC A549 cells by inducing apoptosis and inhibiting proinflammatory cytokines (IL-1 $\beta$, IL-6, IL-8, and TNF- $\alpha$ ) and VEGF, thus inhibiting angiogenesis [64]. A study has shown that luteolin has significant antitumor effects on EGFR with the L858R/ T790M mutation and erlotinib-corrected NSCLC at the organism and cellular levels [65]. Luteolin is also a good antioxidant, scavenging free radicals and producing an antiinflammatory effect [66]. Apigenin and ursolic acid are also good anti-inflammatory and antitumor drugs. Ursolic acid and its derivatives can induce tumor cell apoptosis, inhibit angiogenesis, tumor cell invasion, and metastasis, and can exert an anti-inflammatory effect by inhibiting COX-2 [67]. Apigenin can block the cell cycle in the G1/S and G2/M phases, induce apoptosis, and has anti-inflammation and antioxidation properties [68]. It can inhibit the proliferation of A549 lung cancer cells and the transcriptional activation of VEGF, thus inhibiting tumor neovascularization [69].

At present, the mechanism behind diarrhea as an adverse reaction to EGFR-TKI is not clear. However, some studies have suggested that diarrhea is caused by the decrease of intestinal epithelial growth and repair due to the inhibition of signal transduction in intestinal epithelial cells by EGFRTKI [70]; EGFR-TKI causes the toxicity of the intestinal epithelium and intestinal inflammation, which in turn can increase the exfoliation and apoptosis of the intestinal epithelium [71]. The core compounds identified in this study include several flavonoids, such as quercetin, kaempferol, luteolin, and apigenin. As effective antioxidants, flavonoids have good anti-inflammatory and anticancer activities. Some studies have confirmed a correlation between the intake of flavonoids and lung cancer [72]. Therefore, the core compounds identified in this study can be used as potential therapeutic drugs for lung cancer, and their anti-inflammatory activities can provide a pharmacological reference for alleviating the adverse reaction of diarrhea to EGFR-TKI.

4.3. Molecular Docking. The results of molecular docking activity showed that in the target-compound-TCM network, the highest binding activity was between nobiletin and ESR2. Nobiletin, as a flavonoid compound contained in the TCM citrus peel, has good anticancer and antioxidant pharmacological effects. Nobiletin and its main metabolites, such as $4^{\prime}$-demethylnobiletin and $3^{\prime}$-desmethylnobiletin, can significantly inhibit the occurrence of lung cancer in mice by blocking the cell cycle and inducing apoptosis [73]. Nobiletin combined with paclitaxel, carboplatin, and other chemotherapeutic drugs can significantly inhibit the growth of subcutaneous A549 tumor xenotransplants in mice and enhance the sensitivity of A549 cells to chemotherapeutic drugs by regulating various signaling pathways such as the Akt/GSK3/MYCN pathway $[74,75]$. Nobiletin's good antiinflammatory activity can alleviate the occurrence of diarrhea. Related studies have shown that nobiletin can protect the intestinal epithelium barrier by reducing inflammatory cytokines and can reduce duodenal retraction to treat 
inflammatory bowel disease by inhibiting tumor necrosis factor- $\alpha$ (TNF- $\alpha)$ and the expression of COX-2. It thus has potential intestinal protective function $[76,77]$.

In the past, there were few studies on the relationship between ESR2 and lung cancer. It is also widely believed that ESR2 is closely related to the occurrence and development of breast cancer [78]. However, studies have also found that ESR2 may be a new therapeutic target for lung adenocarcinoma in the future, as its downregulation reduces matrix metallopeptidase 2 (MMP-2) and MMP-9 expression, inhibiting the progression of lung adenocarcinoma through the MEK/ERK signaling pathway [79]. From the above, it can be seen that the targets and related compounds identified in this study have antitumor effects and can relieve the adverse reaction of diarrhea.

The target-compound-TCM network contains 117 target and compound docking combinations, including many compounds with good pharmacological activity, such as palmatine and berberine, which have high docking scores. Palmatine has anticancer, antioxidant, anti-inflammatory, neuroprotective, antibacterial, antiviral, and other pharmacological effects [80]. Berberine is used clinically to treat a variety of diseases with good pharmacological activity; it not only inhibits the proliferation, invasion, and metastasis of cancer cells but also has an excellent inhibitory effect on toxins and bacteria, including Helicobacter pylori. Its ability to protect the intestinal epithelial barrier from injury is used in the treatment of a variety of digestive system diseases [81]. The results of molecular docking showed that the selected compounds and the drug loperamide had good docking activity with their targets, with many compounds having a docking score higher than that of loperamide. It showed that the compounds selected in this study have good medicinal reference value, and the results of molecular docking can provide related compound and target references for the future study of good therapeutic drugs that can be combined with EGFR-TKI treatment.

4.4. Core Chinese Medicine. The core Chinese medicines identified through network pharmacology and data mining were mainly acrid and warm, and their meridian tropism mostly covered the liver, spleen, stomach, and lungs. The characteristics of these core Chinese medicines reflect the characteristics of the TCM theory for treating diarrhea by "warming yang and transforming dampness". The theory of TCM believes that diarrhea originates in the spleen, and the transportation of the water in one's diet cannot be separated from the rise and fall of the spleen and stomach and the adjustment of liver gas. The location of lung cancer is mainly the lungs, and the meridian tropism of these core Chinese medicines aligns with the treatment theory of TCM, which provides a theoretical reference for clinical treatment with TCM.

The core TCMs obtained in this study were Yanhusuo, Danshen, Gancao, Wuzhuyu, Huangbo, Yinyanghuo, Gaoliangjiang, and Huangqi. Modern pharmacology has shown that Yanhusuo extracts contain 13-methyl-palmatrubine, which has antitumor activities and can inhibit the proliferation of lung cancer A549 cells by blocking the activation of the EGFR and MAPK signaling pathways [82]. Studies have confirmed that Yanhusuo alkaloid extracts can inhibit the VEGF signaling pathways, thereby producing antiangiogenic effects, and are expected to be used as antiangiogenic drugs [83]. Yanhusuo extracts also contain berberine, which not only inhibits the proliferation of cancer cells [84] but also can be used in the treatment of inflammatory bowel disease by protecting the intestinal epithelial barrier, regulating the transcription of intestinal inflammatory cytokines, and reducing proinflammatory cytokines such as TNF- $\alpha$, IL-13, IL-6, IL-8, and IFN- $\gamma$ [85]. Therefore, the core Chinese medicine Yanhusuo, when combined with EGFR-TKI treatment, not only has an antitumor effect but also reduces the incidence of diarrhea.

Other selected Chinese medicines also have such effects. The extract of Danshen contains cryptotanshinone, which can inhibit the proliferation of NSCLC cells through the miR-146a-5p/EGFR axis, block the G0/G1 phase of the cell cycle, and promote apoptosis [86, 87]. Tanshinone IIA combined with cisplatin treatment for NSCLC can prevent cancer cell migration and invasion, block the cell cycle in the S phase, and induce A549 cell apoptosis [88]. Gancao, as a widely used TCM, contains a variety of flavonoid compounds and has a variety of pharmaceutical effects, such as antioxidative, anti-inflammatory, antibacterial, antitussive, antiviral, anticancer, and antimutagenic effects [89]. Evodiamine, found in the extract of Wuzhuyu, can inhibit the proliferation of cancer cells through various methods, such as blocking the cell cycle and inhibiting the proliferation of A549 cells. It can induce protective autophagy inf Lewis lung carcinoma cells and has good anticancer activity against many kinds of cancer cells [90]. Evodiamine can also effectively inhibit excessive gastrointestinal motility, providing a basis for the treatment of adverse reactions of diarrhea [91]. In summary, the core Chinese medicines screened in this study are closely related to antitumor effects and can alleviate adverse reactions. Their good pharmacological activity provides a reference for the mining and utilization of related TCMs and provides a theoretical basis for related experimental research and the better use of TCM combined with EGFR-TKI in the treatment of NSCLC in the future.

\section{Conclusion}

In this study, using network pharmacology and data mining, the role of TCM in alleviating EGFR-TKI-associated diarrhea was explored; core targets, compounds, and TCMs were screened using the relevant topological analysis parameters, and the pharmacological activities of the included targets and compounds were further evaluated by molecular docking. Core targets such as OPRM1, OPRD1, and ESR1 were screened, and the related targets of rare adverse reactions such as SCN5A were identified. The core components selected included $\beta$-sitosterol, quercetin, kaempferol, and stigmasterol. The core TCMs selected included Yanhusuo, Danshen, Gancao, and Wuzhuyu. Not only were the relevant targets and compounds consistent with existing research, but also the meridian tropism covered by the core 
TCMs selected was consistent with the theory of TCM. These results can provide data support for the study of the antitumor effect of TCMs and their potential in alleviating diarrhea as an adverse reaction to EGFR-TKI treatment in the future and provide a basis for exploring TCM combined with EGFR-TKI in the treatment of NSCLC.

The advantage of this study is that the relevant data were obtained from an authoritative database. However, a disadvantage is that some of the data may be outdated. Another disadvantage is that the research results depend on the calculation of the software used. There are also certain limitations as the contents of each component of the TCMs are not exactly defined. The results still need to be verified by subsequent animal and clinical trials.

\section{Data Availability}

The datasets supporting the conclusions of this study are included within the article.

\section{Conflicts of Interest}

All authors declare no conflicts of interest.

\section{Authors' Contributions}

HSH and DWC were involved in the design of the study. Data collection and analysis are done by $\mathrm{HSH}$ and DWC together, and the pictures in this article are drawn. The article was jointly drafted by HSH and DWC. ZT and GXY review the data of the article one by one to make sure the data is correct. HW, HP, and LJL provided critical comments and revised the manuscript. All the authors read and approved the final version of the manuscript.

\section{Acknowledgments}

The authors sincerely thank Zheng Li (China Academy of Chinese Medical Sciences) for his contribution to the data analysis of this study. This study was supported by the project Priority Theme Setting and Evidence-Based Research Scheme Design of Traditional Chinese Medicine for $\mathrm{Ma}$ lignant Tumor (K858).

\section{References}

[1] J. R. Molina, P. Yang, S. D. Cassivi, S. E. Schild, and A. A. Adjei, "Non-small cell lung cancer: epidemiology, risk factors, treatment, and survivorship," Mayo Clinic Proceedings, vol. 83, no. 5, pp. 584-594, 2008.

[2] R. S. Herbst, D. Morgensztern, and C. Boshoff, "The biology and management of non-small cell lung cancer," Nature, vol. 553, no. 7689, pp. 446-454, 2018.

[3] O. Kawano, H. Sasaki, K. Endo et al., "PIK3CA mutation status in Japanese lung cancer patients," Lung Cancer, vol. 54, no. 2, pp. 209-215, 2006.

[4] A. Harandi, A. S. Zaidi, A. M. Stocker, and D. A. Laber, "Clinical efficacy and toxicity of anti-EGFR therapy in common cancers," Journal of Oncology, vol. 2009, Article ID 567486, , 2009.
[5] R. Califano, N. Tariq, and S. Compton, "Expert consensus on the management of adverse events from EGFR tyrosine kinase inhibitors in the UK," 2015.

[6] Chinese Society, "EGFR-TKI ADR management Chinese expert consensus," Chinese Journal of Lung Cancer, vol. 22, no. 2, pp. 57-81, 2019.

[7] S. Chen, A. Flower, A. Ritchie et al., "Oral Chinese herbal medicine (CHM) as an adjuvant treatment during chemotherapy for non-small cell lung cancer: a systematic review," Lung Cancer, vol. 68, no. 2, pp. 137-145, 2010.

[8] J. Gao, W. A. Morgan, A. Sanchez-Medina, and O. Corcoran, "The ethanol extract of Scutellaria baicalensis and the active compounds induce cell cycle arrest and apoptosis including upregulation of p53 and Bax in human lung cancer cells," 2011.

[9] A. L. Hopkins, "Network pharmacology: the next paradigm in drug discovery," Nature Chemical Biology, vol. 4, no. 11, pp. 682-690, 2008.

[10] D. Yan and X. Xiao, "Investigation on pattern and methods of quality control for Chinese materia medica based on dao-di herbs and bioassay-bioassay for Coptis chinensis," 2011.

[11] D. Gfeller, O. Michielin, and V. Zoete, "Shaping the interaction landscape of bioactive molecules," Bioinformatics, vol. 29, no. 23, pp. 3073-3079, 2013.

[12] A. Daina, O. Michielin, and V. Zoete, "Swiss target prediction: updated data and new features for efficient prediction of protein targets of small molecules," Nucleic Acids Research, vol. 47, no. W1, pp. W357-W364, 2019.

[13] M. Safran, I. Dalah, J. Alexander et al., "GeneCards Version 3: the human gene integrator," Database, vol. 2010, p. baq020, 2010.

[14] J. S. Amberger, C. A. Bocchini, F. Schiettecatte, A. F. Scott, and A. Hamosh, "OMIM.org: online Mendelian Inheritance in Man (OMIM), an online catalog of human genes and genetic disorders," Nucleic Acids Research, vol. 43, no. D1, pp. D789-D798, 2015.

[15] J. Piñero, J. M. Ramírez-Anguita, J. Saüch-Pitarch et al., "The DisGeNET knowledge platform for disease genomics: 2019 update," Nucleic Acids Research, vol. 48, no. D1, pp. D845-D855, 2020.

[16] D. S. Wishart, Y. D. Feunang, A. C. Guo et al., "DrugBank 5.0: a major update to the DrugBank database for 2018," Nucleic Acids Research, vol. 46, no. D1, pp. D1074-D1082, 2018.

[17] T. UniProt Consortium, "UniProt: the universal protein knowledgebase," Nucleic Acids Research, vol. 46, no. 5, p. 2699, 2018.

[18] J. Ru, P. Li, and J. Wang, "TCMSP: a database of systems pharmacology for drug discovery from herbal medicines," Journal of Cheminformatics, vol. 6, p. 13, 2014.

[19] X. Xu, W. Zhang, C. Huang et al., "A novel chemometric method for the prediction of human oral bioavailability," International Journal of Molecular Sciences, vol. 13, no. 6, pp. 6964-6982, 2012.

[20] D. F. Veber, S. R. Johnson, H.-Y. Cheng, B. R. Smith, K. W. Ward, and K. D. Kopple, "Molecular properties that influence the oral bioavailability of drug candidates," Journal of Medicinal Chemistry, vol. 45, no. 12, pp. 2615-2623, 2002.

[21] W. Tao, X. Xu, X. Wang et al., "Network pharmacology-based prediction of the active ingredients and potential targets of Chinese herbal Radix Curcumae formula for application to cardiovascular disease," Journal of Ethnopharmacology, vol. 145, no. 1, pp. 1-10, 2013.

[22] P. Ertl, B. Rohde, and P. Selzer, "Fast calculation of molecular polar surface area as a sum of fragment-based contributions 
and its application to the prediction of drug transport properties," Journal of Medicinal Chemistry, vol. 43, no. 20, pp. 3714-3717, 2000.

[23] P. Shannon, A. Markiel, and O. Ozier, "Cytoscape: a software environment for integrated models of biomolecular interaction networks," Genome Research, vol. 13, no. 11, pp. 2498-2504, 2003.

[24] C. P. Commission, Pharmacopoeia of People's Republic of China (2015 Edition): 北京, China Medical Science Press, Beijing, China, 2015.

[25] G. Zhong, Chinese Medicine (New Century): 北 京, China Press of Traditional Chinese Medicine, Beijing, China, 4th edition, 2016.

[26] M. Yang, J. Chen, L. Xu, and G. Ji, "Navigating traditional Chinese medicine network pharmacology and computational tools," Evidence-Based Complementary And Alternative Medicine : ECAM, vol. 2013, Article ID 731969, 2013.

[27] K. Hsin, S. Ghosh, and H. Kitano, "Combining machine learning systems and multiple docking simulation packages to improve docking prediction reliability for network pharmacology," PLoS One, vol. 8, no. 12, Article ID e83922, 2013.

[28] J. Greenhalgh, K. Dwan, and A. Boland, "First-line treatment of advanced epidermal growth factor receptor (EGFR) mutation positive non-squamous non-small cell lung cancer," The Cochrane Database of Systematic Reviews, vol. 5, Article ID D10383, 2016.

[29] B. Han, S. Tjulandin, K. Hagiwara et al., "EGFR mutation prevalence in Asia-Pacific and Russian patients with advanced NSCLC of adenocarcinoma and non-adenocarcinoma histology: the IGNITE study," Lung Cancer, vol. 113, pp. 37-44, 2017.

[30] K. E. Barrett, "Epithelial transport in digestive diseases - mice, monolayers and mechanisms: 2019 hans using distinguished lecture article," American Journal of Physiology. Cell Physiology, vol. 318, no. 6, 2020.

[31] H. S. Rugo, J. A. Di Palma, D. Tripathy et al., "The characterization, management, and future considerations for ErbBfamily TKI-associated diarrhea," Breast Cancer Research and Treatment, vol. 175, no. 1, pp. 5-15, 2019.

[32] L. Jiao, J. Xu, and J. Sun, "Chinese herbal medicine combined with EGFR-TKI in EGFR mutation-positive advanced pulmonary adenocarcinoma (CATLA): a multicenter, randomized, double-blind, placebo-controlled trial," Frontiers in Pharmacology, vol. 10, p. 732, 2019.

[33] M. Tang, S. Wang, and B. Zhao, "Traditional Chinese medicine prolongs progression-free survival and enhances therapeutic effects in epidermal growth factor receptor tyrosine kinase inhibitor (EGFR-TKI) treated non-small-cell lung cancer (NSCLC) patients harboring EGFR mutations," Medical Science Monitor International Medical Journal of Experimental And Clinical Research, vol. 25, no. 614, pp. 8430-8437, 2019.

[34] Z.-l. Liu, W.-r. Zhu, W.-c. Zhou et al., "Traditional Chinese medicinal herbs combined with epidermal growth factor receptor tyrosine kinase inhibitor for advanced non-small cell lung cancer: a systematic review and meta-analysis," Journal of Integrative Medicine, vol. 12, no. 4, pp. 346-358, 2014.

[35] G. W. Pasternak and Y.-X. Pan, "Mu opioids and their receptors: evolution of a concept," Pharmacological Reviews, vol. 65, no. 4, pp. 1257-1317, 2013.

[36] A. Jarmuż, M. Zielińska, K. Leń, M. Banaszek, M. Storr, and J. Fichna, "Review: the role of MOP and DOP receptors in treatment of diarrheapredominant irritable bowel syndrome,"
Mini Reviews in Medicinal Chemistry, vol. 16, no. 18, pp. 1462-1469, 2016.

[37] K. J. Lee, "Pharmacologic agents for chronic diarrhea," Intestinal Research, vol. 13, no. 4, pp. 306-312, 2015.

[38] M. Corsetti and P. Whorwell, "New therapeutic options for IBS: the role of the first in class mixed $\mu$ - opioid receptor agonist and $\delta$-opioid receptor antagonist (mudelta) eluxadoline," Expert Review of Gastroenterology \& Hepatology, vol. 11, no. 4, pp. 285-292, 2017.

[39] J. Pannemans and M. Corsetti, "Opioid receptors in the GI tract: targets for treatment of both diarrhea and constipation in functional bowel disorders?" Current Opinion in Pharmacology, vol. 43, pp. 53-58, 2018.

[40] F. J. Ehlert, R. S. Ostrom, and G. W. Sawyer, "Subtypes of the muscarinic receptor in smooth muscle," Life Sciences, vol. 61, no. 18, pp. 1729-1740, 1997.

[41] A. Atmaca, S.-E. Al-Batran, R. M. Wirtz et al., "The validation of estrogen receptor $1 \mathrm{mRNA}$ expression as a predictor of outcome in patients with metastatic non-small cell lung cancer," International Journal of Cancer, vol. 134, no. 10, pp. 2314-2321, 2014.

[42] R. Pietras, D. Márquez, H. Chen, E. Tsai, O. Weinberg, and M. Fishbein, "Estrogen and growth factor receptor interactions in human breast and non-small cell lung cancer cells," Steroids, vol. 70, no. 5-7, pp. 372-381, 2005.

[43] J. Y. Song, J. M. Siegfried, B. Diergaarde et al., "Genetic variation in ESR2 and estrogen receptor-beta expression in lung tumors," Cancer Epidemiology, vol. 37, no. 4, pp. 518-522, 2013.

[44] S.-H. I. Ou, "Republished: lung cancer in never-smokers. does smoking history matter in the era of molecular diagnostics and targeted therapy?" Postgraduate Medical Journal, vol. 90, no. 1062 , pp. 228-235, 2014.

[45] A. A. M. Wilde and A. S. Amin, "Clinical spectrum of SCN5A mutations," JACC: Clinical Electrophysiology, vol. 4, no. 5, pp. 569-579, 2018.

[46] M. Schiefer, L. E. L. Hendriks, T. Dinh, U. Lalji, and A. C. Dingemans, "Current perspective: osimertinib-induced QT prolongation: new drugs with new side-effects need careful patient monitoring," 2018.

[47] T. S. Mok, Y. Wu, and M. Ahn, "Osimertinib or platinumpemetrexed in EGFR T790M-positive lung cancer," 2017.

[48] A. Leone, A. Spada, A. Battezzati, A. Schiraldi, J. Aristil, and S. Bertoli, "Moringa oleifera seeds and oil: characteristics and uses for human health," International Journal of Molecular Sciences, vol. 17, no. 12, 2016.

[49] M. S. Bin Sayeed and S. S. Ameen, "Beta-sitosterol: a promising but orphan nutraceutical to fight against cancer," Nutrition and Cancer, vol. 67, no. 8, pp. 1214-1220, 2015.

[50] T. Rajavel, R. Mohankumar, G. Archunan, K. Ruckmani, and K. P. Devi, "Beta sitosterol and Daucosterol (phytosterols identified in Grewia tiliaefolia) perturbs cell cycle and induces apoptotic cell death in A549 cells," Scientific Reports, vol. 7, no. 1, p. 3418, 2017.

[51] D. Kashyap, S. Mittal, K. Sak, P. Singhal, and H. S. Tuli, "Molecular mechanisms of action of quercetin in cancer: recent advances," Tumor Biology, vol. 37, no. 10, pp. 12927-12939, 2016.

[52] X. Zhao and J. Zhang, "Mechanisms for quercetin in prevention of lung cancer cell growth and metastasis," Zhong nan da xue xue bao. Yi xue ban = Journal of Central South University. Medical sciences, vol. 40, no. 6, pp. 592-597, 2015.

[53] S. H. Lee, E. J. Lee, K. H. Min et al., "Quercetin enhances chemosensitivity to gemcitabine in lung cancer cells by 
inhibiting heat shock protein 70 expression," Clinical Lung Cancer, vol. 16, no. 6, pp. e235-e243, 2015.

[54] A. Massi, O. Bortolini, and D. Ragno, "Research progress in the modification of quercetin leading to anticancer agents," Molecules (Basel, Switzerland), vol. 22, no. 8, 2017.

[55] T. Birdi, P. Daswani, S. Brijesh, P. Tetali, A. Natu, and N. Antia, "Newer insights into the mechanism of action of Psidium guajava L. leaves in infectious diarrhoea," $B M C$ Complementary and Alternative Medicine, vol. 10, no. 671, p. 33, 2010.

[56] G. D'Andrea, "Quercetin: a flavonol with multifaceted therapeutic applications?” Fitoterapia, vol. 106, pp. 256-271, 2015.

[57] W.-W. Huang, S.-C. Tsai, S.-F. Peng et al., "Kaempferol induces autophagy through AMPK and AKT signaling molecules and causes G2/M arrest via downregulation of CDK1/ cyclin B in SK-HEP-1 human hepatic cancer cells," International Journal of Oncology, vol. 42, no. 6, pp. 2069-2077, 2013.

[58] A. Y. Chen and Y. C. Chen, "A review of the dietary flavonoid, kaempferol on human health and cancer chemoprevention," Food Chemistry, vol. 138, no. 4, pp. 2099-2107, 2013.

[59] H. Luo, G. O. Rankin, N. Juliano, B.-H. Jiang, and Y. C. Chen, "Kaempferol inhibits VEGF expression and in vitro angiogenesis through a novel ERK-NF $\kappa$ B-cMyc-p21 pathway," Food Chemistry, vol. 130, no. 2, pp. 321-328, 2012.

[60] M. L. Ackland, S. van de Waarsenburg, and R. Jones, "Synergistic antiproliferative action of the flavonols quercetin and kaempferol in cultured human cancer cell lines," In Vivo (Athens, Greece), vol. 19, no. 1, pp. 69-76, 2005.

[61] K. P. Devi, D. S. Malar, S. F. Nabavi et al., "Kaempferol and inflammation: from chemistry to medicine," Pharmacological Research, vol. 99, pp. 1-10, 2015.

[62] K. Li, D. Yuan, R. Yan, L. Meng, Y. Zhang, and K. Zhu, "Stigmasterol exhibits potent antitumor effects in human gastric cancer cells mediated via inhibition of cell migration, cell cycle arrest, mitochondrial mediated apoptosis and inhibition of JAK/STAT signalling pathway," Journal of B.U.ON. : Official Journal of the Balkan Union of Oncology, vol. 23, no. 5, pp. 1420-1425, 2018.

[63] S. Feng, Z. Dai, A. Liu et al., “ $\beta$-Sitosterol and stigmasterol ameliorate dextran sulfate sodium-induced colitis in mice fed a high fat Western-style diet," Food \& Function, vol. 8, no. 11, pp. 4179-4186, 2017.

[64] M. Imran, A. Rauf, and T. Abu-Izneid, "Luteolin, a flavonoid, as an anticancer agent: a review," Biomedicine \& Pharmacotherapy = Biomedecine \& Pharmacotherapie, vol. 112, Article ID 108612, 2019.

[65] L. Bai, Y. Nong, Y. Shi et al., "Luteolin inhibits hepatitis B virus replication through extracellular signal-regulated kinase-mediated down-regulation of hepatocyte nuclear factor $4 \alpha$ expression," Molecular Pharmaceutics, vol. 13, no. 2, pp. 568-577, 2016.

[66] G. Seelinger, I. Merfort, and C. Schempp, "Anti-oxidant, antiinflammatory and anti-allergic activities of luteolin," Planta Medica, vol. 74, no. 14, pp. 1667-1677, 2008.

[67] S. Mlala, A. O. Oyedeji, M. Gondwe, and O. O. Oyedeji, "Ursolic acid and its derivatives as bioactive agents," Molecules (Basel, Switzerland), vol. 24, no. 15, 2019.

[68] A. Kowalczyk, A. Bodalska, M. Miranowicz, and K. Karłowicz-Bodalska, "Insights into novel anticancer applications for apigenin," Advances in Clinical and Experimental Medicine, vol. 26, no. 7, pp. 1143-1146, 2017.

[69] Z.-D. Li, L.-Z. Liu, X. Shi, J. Fang, and B.-H. Jiang, "Benzo[a] pyrene-3,6-dione inhibited VEGF expression through inducing HIF-1 $\alpha$ degradation," Biochemical And Biophysical Research Communications, vol. 357, no. 2, pp. 517-523, 2007.

[70] J. M. Bowen, "Mechanisms of TKI-induced diarrhea in cancer patients," Current Opinion in Supportive \& Palliative Care, vol. 7, no. 2, pp. 162-167, 2013.

[71] L. Vereecke, R. Beyaert, and G. van Loo, "Enterocyte death and intestinal barrier maintenance in homeostasis and disease," Trends in Molecular Medicine, vol. 17, no. 10, pp. 584-593, 2011.

[72] P. Knekt, R. Järvinen, R. Seppänen et al., "Dietary flavonoids and the risk of lung cancer and other malignant neoplasms," American Journal of Epidemiology, vol. 146, no. 3, pp. 223230, 1997.

[73] Y. Sun, Y. Han, M. Song et al., "Inhibitory effects of nobiletin and its major metabolites on lung tumorigenesis," Food \& Function, vol. 10, no. 11, pp. 7444-7452, 2019.

[74] S. Uesato, H. Yamashita, R. Maeda et al., "Synergistic antitumor effect of a combination of paclitaxel and carboplatin with nobiletin from Citrus depressa on non-small-cell lung cancer cell lines," Planta Medica, vol. 80, no. 06, pp. 452-457, 2014.

[75] J. Y. Moon, L. V. Manh Hung, T. Unno, and S. K. Cho, "Nobiletin enhances chemosensitivity to adriamycin through modulation of the akt/GSK3 $\beta / \beta^{-}$catenin/MYCN/MRP1 signaling pathway in A549 human non-small-cell lung cancer cells," Nutrients, vol. 10, no. 12, 2018.

[76] X. Wen, H. Zhao, L. Wang et al., "Nobiletin attenuates DSSinduced intestinal barrier damage through the HNF4 $\alpha$ claudin-7 signaling pathway," Journal of Agricultural and Food Chemistry, vol. 68, no. 16, pp. 4641-4649, 2020.

[77] W. He, Y. Li, and M. Liu, "Citrus aurantium L. and its flavonoids regulate TNBS-induced inflammatory bowel disease through anti-inflammation and suppressing isolated jejunum contraction," International Journal of Molecular Sciences, vol. 19, no. 10, 2018.

[78] Z. Dai, T. Tian, and M. Wang, "Genetic polymorphisms of estrogen receptor genes are associated with breast cancer susceptibility in Chinese women," Cancer Cell International, vol. 19, p. 11, 2019.

[79] W. Chen, B. Xin, H. Pang et al., "Downregulation of estrogen receptor $\beta$ inhibits lung adenocarcinoma cell growth," Oncology Reports, vol. 41, no. 5, pp. 2967-2974, 2019.

[80] J. Long, J. Song, L. Zhong, Y. Liao, L. Liu, and X. Li, "Palmatine: a review of its pharmacology, toxicity and pharmacokinetics,” Biochimie, vol. 162, pp. 176-184, 2019.

[81] D. Song, J. Hao, and D. Fan, "Biological properties and clinical applications of berberine," Frontiers of Medicine, vol. 14, no. 5, pp. 564-582, 2020.

[82] J. Chen, X. Lu, C. Lu et al., "13-Methyl-palmatrubine induces apoptosis and cell cycle arrest in A549 cells in vitro and in vivo," Oncology Reports, vol. 36, no. 5, pp. 2526-2534, 2016.

[83] L. Wan, Y. Zhao, and Q. Zhang, "Alkaloid extract of Corydalis yanhusuo inhibits angiogenesis via targeting vascular endothelial growth factor receptor signaling," BMC Complementary and Alternative Medicine, vol. 19, no. 1, p. 359, 2019.

[84] L. M. G. Ortiz, P. Lombardi, M. Tillhon, and A. I. Scovassi, "Berberine, an epiphany against cancer," Molecules (Basel, Switzerland), vol. 19, no. 8, pp. 12349-12367, 2014.

[85] K. Zou, Z. Li, Y. Zhang et al., "Advances in the study of berberine and its derivatives: a focus on anti-inflammatory and anti-tumor effects in the digestive system," Acta Pharmacologica Sinica, vol. 38, no. 2, pp. 157-167, 2017.

[86] P. Qi, Y. Li, X. Liu et al., "Cryptotanshinone suppresses nonsmall cell lung cancer via microRNA-146a-5p/EGFR axis," 
International Journal of Biological Sciences, vol. 15, no. 5, pp. 1072-1079, 2019.

[87] S. Kim, O. Kang, and D. Kwon, "Cryptotanshinone induces cell cycle arrest and apoptosis of NSCLC cells through the PI3K/Akt/GSK-3 $\beta$ pathway," International Journal of Molecular Sciences, vol. 19, no. 9, 2018.

[88] X. Z. Liao, Y. Gao, S. Huang et al., "Tanshinone IIA combined with cisplatin synergistically inhibits non-small-cell lung cancer in vitro and in vivo via down-regulating the phosphatidylinositol 3-kinase/Akt signalling pathway," Phytotherapy Research, vol. 33, no. 9, pp. 2298-2309, 2019.

[89] G. Pastorino, L. Cornara, S. Soares, F. Rodrigues, and M. B. P. P. Oliveira, "Liquorice (Glycyrrhiza glabra): a phytochemical and pharmacological review," Phytotherapy Research, vol. 32, no. 12, pp. 2323-2339, 2018.

[90] X. Hu, D. Li, and C. Chu, "Antiproliferative effects of alkaloid evodiamine and its derivatives," International Journal of Molecular Sciences, vol. 19, no. 11, 2018.

[91] H. X. Ren, Q. C. Tang, L. Yan, H. Xia, and H. S. Luo, "Evodiamine inhibits gastrointestinal motility via CCK and CCK1 receptor in water-avoidence stress rat model," Life Sciences, vol. 209, pp. 210-216, 2018. 